\title{
Padrão de Desenho para a Família de Grafos Gêmeos
}

\author{
Cassio Ferreira Merlo ${ }^{1}$, Mauro Pinheiro Rodrigues ${ }^{1}$, Marcia Helena Moreira Paiva ${ }^{2}$ \\ ${ }^{1}$ Laboratório de Projetos em Design (ProDesignUfes) \\ Universidade Federal do Espírito Santo (UFES) -Vitória - ES - Brasil \\ ${ }^{2}$ Laboratório de Telecomunicações (LabTel) \\ Universidade Federal do Espírito Santo (UFES) - Vitória - ES - Brasil \\ cassiofmerlo@gmail.com, \{mauro.pinheiro,marcia.paiva\}@ufes.br
}

\begin{abstract}
This paper is about the critical thinking involved in the development of a graphic pattern for the family of twin graphs, considering as its main objective the characterization of the family, and the ease of design. This work is based on the search for developing a visual pattern for this purpose, starting from empty until the final form.
\end{abstract}

Resumo. Este artigo trata da reflexão crítica envolvida no desenvolvimento de um padrão gráfico para a família de grafos gêmeos, considerando como seu objetivo principal a caracterização da família e a facilidade de concepção. Este trabalho é baseado na busca pelo desenvolvimento de um padrão visual para tal propósito, partindo do vazio até a forma final.

\section{Introdução}

Um grafo $G=(V, A)$ é uma estrutura consistindo em um conjunto de vértices $V$ e um conjunto de arestas $A$, onde os vértices, que também podem ser chamados de nós, são pontos distribuídos no espaço, e as arestas são linhas representando relações entre pares de vértices. Em outras palavras, grafos são conjuntos tais que sua representação geométrica se dá pela relação entre vértices e arestas, de forma que as arestas estejam conectadas em função de relações entre os vértices.

Neste trabalho buscou-se, a princípio, identificar e estudar as características de grafos, particularmente grafos gêmeos, estudando-se a viabilidade de uma representação padrão para esta família de grafos, que possui diversas características interessantes para problemas de roteamento com proteção e, em particular, para o projeto de redes de telecomunicações [PAIVA et al. 2013]. Redes de telecomunicações devem atender certos requisitos como confiabilidade, desempenho e custo. A partir da modelagem via grafos, é possível definir as características topológicas de uma rede de telecomunicações, traduzidas em parâmetros provenientes da teoria de grafos, tais como diâmetro, grau máximo e conectividade. Em geral usa-se ferramentas computacionais tais como yEd Graph Editor ${ }^{1}$ ou NetworkX ${ }^{2}$ para desenho automático de grafos. Entretanto, nem sempre o desenho do grafo obtido por estas ferramentas permite a identificação imediata das características topológicas da rede de telecomunicações associada. Nesse contexto, este trabalho busca desenvolver uma representação padrão para os grafos gêmeos, a exemplo da representação padrão de árvores proposta em [HARARY 1969].

\footnotetext{
${ }^{1}$ www.yworks.com/products/yed

${ }^{2}$ networkx.github.io
} 
Grafos gêmeos são grafos 2-geodesicamente conexos minimais com respeito ao número de arestas [FARLEY e PROSKUROWSKI 1997], ou seja, são grafos que possuem pelo menos duas geodésicas disjuntas (i.e., dois menores caminhos disjuntos) para cada par de vértices não-adjacentes. Todos os grafos gêmeos são bipartidos e planares. Com exceção dos grafos gêmeos, os únicos grafos 2geodesicamente conexos minimais são o $C_{3}$ (ciclo de ordem 3) e o $Q_{3}$ (cubo de ordem 8) [FARLEY e PROSKUROWSKI 1997].

A partir de reflexões sobre essas características, foi possível entender que essas propriedades deveriam ser preservadas na concepção de um modelo visual. Ao mesmo tempo foi considerado que a facilidade de identificação e leitura do grafo deveriam ser uma característica, auxiliando assim o usuário no reconhecimento do mesmo.

\section{Primeiros esboços}

Utilizando-se do processo de resolução de problemas proposto em [LOBACK 2001], decidiu-se iniciar tal desenvolvimento por meio de esboços manuais, seguido de estudos e desenhos com o programa yEd Graph Editor. A partir dessa decisão, realizou-se um levantamento gráfico de grafos gêmeos existentes, que apresentassem características semelhantes às encontradas anteriormente, mas com desenhos diferentes. Foram encontrados alguns poucos desenhos publicados no artigo [PAIVA et al. 2013], um dos quais é reproduzido na Figura 1. Esses primeiros desenhos serviram como início para os primeiros estudos e esboços de um modelo adequado de visualização dos grafos.

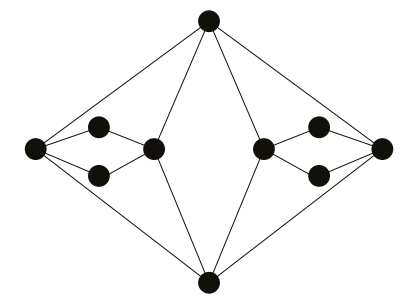

Figura 1. Desenho inicial encontrado em [PAIVA et al. 2013].

Simultaneamente aos desenhos iniciais, foi observado como o grafo foi percebido por um grupo de usuários, principalmente como sua visualização espacial e fisionomia são agradáveis na percepção e apesar de apresentar parcialmente uma boa aparência, o principal fator negativo observado foi a complexidade para a construção do grafo a partir do vazio, tornando a tarefa complicada e demorada. Concluiu-se então, que a adoção desse aspecto visual, tornar-se-ia mais trabalhosa na disseminação e construção de um modelo útil, necessitando então incluir como característica a simplicidade e rapidez na concepção estrutural do grafo.

\section{Desenvolvimento}

Para demonstração da construção do novo padrão visual, utilizamos como base o primeiro grafo encontrado no artigo [PAIVA et al. 2013]. Primeiramente, precisamos observar e analisar o grafo quanto a quantidade de vértices e o grau de cada um, ou seja, a quantidade de ligações de cada um. Na Figura 2 observamos que o grafo é composto por 10 vértices, sendo 4 vértices com 2 ligações e 6 vértices com 4 ligações. A partir dessa informação é possível construir o grafo no novo padrão. 


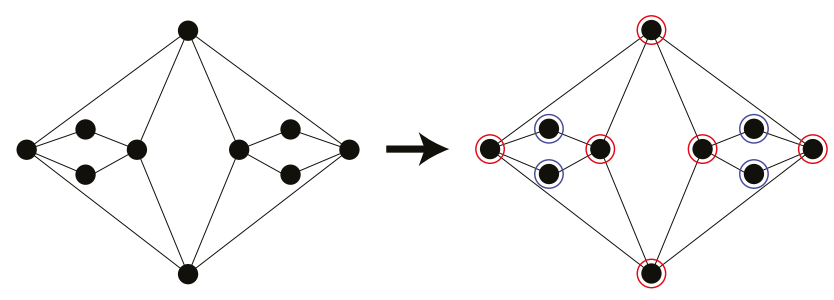

Figura 2. Primeira análise, observando-se os graus dos vértices.

Para a construção do novo modelo, primeiramente divide-se o número de vértices por dois; no caso do grafo que estamos usando, obtemos o valor cinco. Em seguida, desenha-se um quadrado com 4 vértices. Os dois vértices superiores levam os dois primeiros números sequenciais ( 1 e 2, no caso), e os vértices inferiores levaram os dois primeiros números sequenciais a partir da metade do número de vértices ( 6 e 7 , no caso). Ao mesmo tempo, determinou-se um padrão onde os números ímpares são expostos no lado esquerdo e os pares do lado direito. O resultado desse processo é ilustrado na Figura 3.
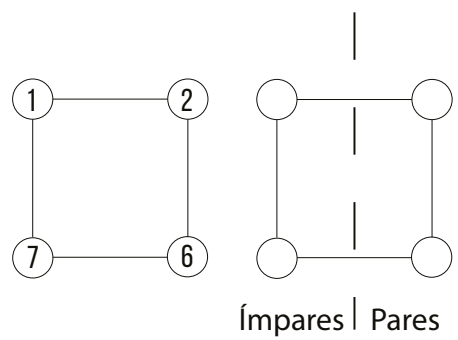

Figura 3. Passo-a-passo inicial.

Dessa forma, é necessário somente organizar os números em sequências. Na parte superior deve estar a primeira metade dos vértices (até o vértice 5), na metade inferior os demais. Após isso, inicialmente é necessário ligar os vértices com lado de menor quantidade de vértices, ao outro lado superior, por exemplo ligar o vértice 2 ao vértice 3 , em seguida ligar ao lado oposto do mesmo, vértice 4, sequencialmente repetiremos o primeiro passo, ligaremos o vértice 4 ao vértice 5. Esses passos são ilustrados na Figura 4. Posteriormente, deve-se fazer o mesmo procedimento com todos os lados do grafo. A Figura 5 ilustra o resultado final obtido por esse procedimento, que corresponde ao novo padrão gráfico proposto a partir do grafo da Figura 1.

(5)

(3)

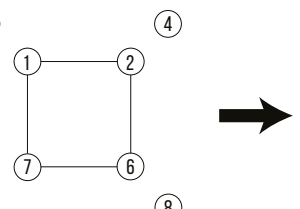

(10)
(5)

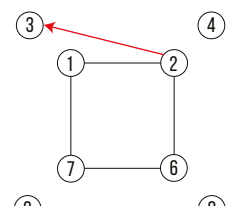

(8)

(10)

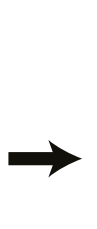

(5)

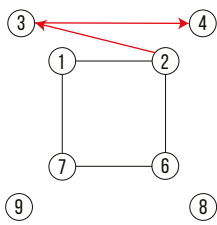

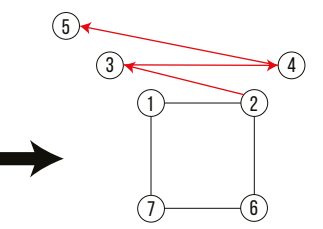

(9)
(8)

(10)

Figura 4. Passo-a-passo final. 


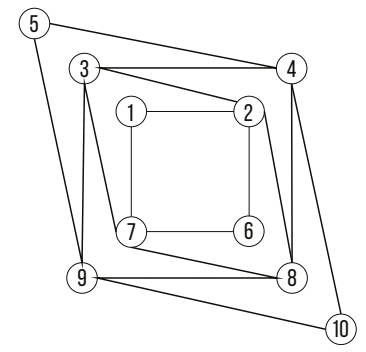

Figura 5. Padrão final de desenho do grafo gêmeo mostrado na Figura 1.

\section{Resultados}

Com o desenvolvimento da pesquisa foi possível observar o processo de construção de um padrão para grafos gêmeos, preservando suas características e sendo adequado às propostas a serem desenvolvidas. Além disso, a pesquisa levou à criação de um modelo final de padrão gráfico a ser explorado pelos usuários. Tal produto servirá como insumo para novos estudos e pesquisas, bem como ampliará o acervo gráfico de grafos gêmeos, se tornando patrimônio público imaterial da comunidade científica.

Como continuação deste trabalho, estamos elaborando um algoritmo para o desenho automático de grafos gêmeos, a partir do método aqui apresentado. Simultaneamente com o insumo produzido, a pesquisa apresenta um processo metodológico que pode ser utilizado em outros casos semelhantes, sendo uma contribuição para futuras pesquisas na área de desenvolvimento de padrões para desenho de grafos.
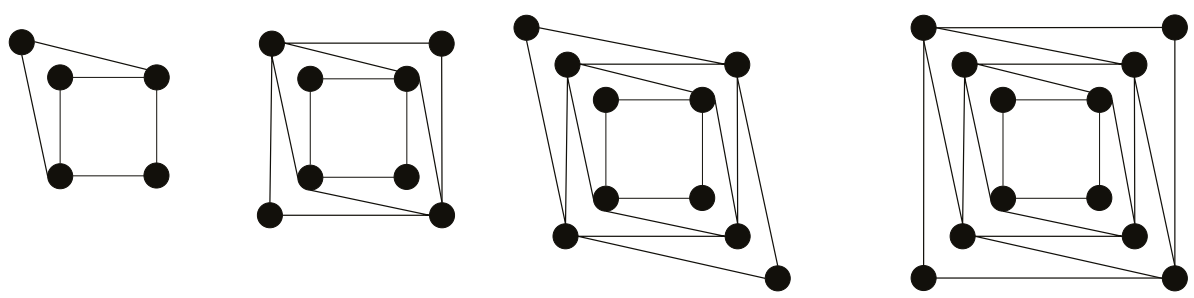

Figura 6. llustrações do novo padrão obtido para o desenho de grafos gêmeos.

\section{Agradecimentos}

Este trabalho é parcialmente financiado pelo CNPq (processo 462477/2014-2) e pela CAPES (processo PNPD20130772).

\section{Referências}

FARLEY, A. e PROSKUROWSKI, A. (1997). Minimum self-repairing graphs. Graphs and Combinatorics, 13:345-351.

HARARY, F. (1969). Graph Theory. Addison-Wesley Series in Mathematics. AddisonWesley Publishing Company.

LOBACK, B. (2001). Design industrial: bases para a configuração dos produtos industriais. Ed. Blucher, São Paulo.

PAIVA, M., CAPOROSSI, G., e SEGATTO, M. (2013). Twin graphs for OTN physical topology design. Les Cahiers du GERAD, 48:1-12. 